\title{
A Study About One Generation of Finite Simple Groups and Finite Groups
}

\author{
Nader Taffach \\ Correspondence: Faculty of Science, Department of Mathematics, Idlib University, Syria.
}

Received: April 5, 2021 Accepted: April 26, 2021 Online Published: May 18, 2021

doi:10.5539/jmr.v13n3p59

URL: https://doi.org/10.5539/jmr.v13n3p59

\begin{abstract}
In this paper, we study the problem of how a finite group can be generated by some subgroups. In order to the finite simple groups, we show that any finite non-abelian simple group can be generated by two Sylow $p_{1}$ - and $p_{2}$-subgroups, where $p_{1}$ and $p_{2}$ are two different primes. We also show that for a given different prime numbers $p$ and $q$, any finite group can be generated by a Sylow $p$-subgroup and a $q$-subgroup.
\end{abstract}

Keywords: Sylow $p$-subgroup, sporadic group, abelian group, classification of groups

MSC2020: 20D05, 20D08, 20D20

\section{Introduction}

Finite groups often arise when considering the symmetry of mathematical or physical objects, when those objects admit just a finite number of structure-preserving transformations. Important examples of finite groups include cyclic groups and permutation groups (Praeger \& Schneider, 2018). During the twentieth century, mathematicians investigated some aspects of the theory of finite groups in great depth. One major area of study has been the classification of groups (Solomon, 2001). At the beginning of the 1980s, the development of the theory of finite groups culminated in the classification of the finite simple groups, an impressive and convincing demonstration of the strength of its methods and results (Kurzweil \& Stellmacher, 2004).

The classification of finite simple groups, (Gorenstein, Richard \& Solomon, 2018), i.e. groups with no nontrivial normal subgroup, was completed in 2004. As a consequence, the complete classification of finite simple groups was achieved, meaning that all those simple groups from which all finite groups can be built are now known (Aschbacher, 2004).

Via its importance, the concept of the generation of a finite simple group and a finite group has widespread attention along time, where generation of finite groups by suitable subsets has many applications to groups and their representations. For example, due to the motivation to study irreducible projective representations of the sporadic simple groups, in (Martino, Pellegrini \& Zalesski, 2014) it was established a useful connection between the generation of groups by conjugate elements and the existence of elements representable by almost cyclic matrices. It is known that finite non-abelian simple groups are 2-generated (Steinberg, 1962). In (Aschbacher \& Guralnick, 1984) authors showed that any sporadic simple group can be generated by an involution and another suitable element, recall that a sporadic group is one of the 26 exceptional groups found in the classification of finite simple groups (Wilson, 1998). In (King, 2017) it was shown that every finite non-abelian simple group is generated by an involution and an element of prime order. In (Burness, Liebeck, \& Shalev, 2013) authors showed that any maximal subgroup of a non-abelian finite simple group is 4-generated or less and that this bound is best possible. In 2007, Slattery in his paper (Slattery, 2007) described computational methods to enumerate, construct and identify finite groups of square-free order. Also, in (Breuer, Guralnick, \& Kantor, 2008) authors conjectured that any finite group is $\frac{3}{2}$-generated if and only if every proper quotient is cyclic. In (Burness, Guralnick, \& Harper, 2021) it was reduced this conjecture to almost simple groups. Recently, in (Dietrich \& Low, 2021) the authors, generalized Slattery's result to the class of finite groups that have cyclic Sylow subgroups and provide an implementation for the computer algebra system GAP (see https://www.gap-system.org/).

In this paper, in order to the finite simple groups, we show that any finite non-abelian simple group can be generated by two Sylow $p_{1}$ - and $p_{2}$-subgroups, where $p_{1}$ and $p_{2}$ are two different primes. Also, we show that for a given different primes $p$ and $q$, every finite group can be generated by a $p$-subgroup and a $q$-subgroup.

The paper is consists of an introduction and two fundamental sections. In Section 2, we study some generations of finite simple groups, we prove three lemmas, from which we conclude the fundamental theorem about the generation of finite simple groups by different Sylow subgroups. In Section 3, we set other fundamental results of the paper, concerning the generation of finite groups by some subgroups. 


\section{Generations of Simple Groups}

For the fundamental result in this section (see Theorem 1, below), we need some auxiliary lemmas. Therefore firstly we will set three lemmas, with their proof.

Lemma 1. Let $S=A l t_{n}, n \geq 5$. If $t \leq n$ is prime, then $S$ can be generated by a Sylow 2 -subgroup and a Sylow $t$-subgroup.

Proof. When $n \leq 8$ the result is clear. Suppose that $n>8$ and is an odd number. Let $R$ be a Sylow 2-subgroup of $S$. The orbits of $R$ have distinct sizes and there are less than $\log _{2} n$ orbits. Let us choose a $t$-element $x$, such that $x$ has a cycle of length $t^{a} \geq \sqrt{n}$ for $t$ odd and at least $n / 2$ if $t=2$. In both cases, the size of the orbit is greater than the number of orbits of $R$. Thus, we can choose a conjugate of $x$ so that an orbit of $x$ intersects every orbit of $R . J:=$ $\langle R, x\rangle$ is transitive. Since $n$ is odd and $R \leq J$, we find that $J$ is primitive. Since $n>8$, the only primitive subgroup of $A l t_{n}$ containing an element moving 4 points is $A l t_{n}$ (Wielandt, 1964), and therefore we reached the result.

Now, suppose that $n>9$ is an even number. From the previous case, i.e. when $n$ is odd, we find that $A l t_{n-1}=\left\langle R_{0}, T\right\rangle$ for some $t$-subgroup $T$ and Sylow 2-subgroup $R_{0}$. Let $R$ be a Sylow 2-subgroup of $S$ properly containing $R_{0}$. Then $\langle R, T\rangle$ properly contains $\left\langle R_{0}, T\right\rangle=A l t_{n-1}$, whence $\langle R, T\rangle=S$. Thus we reached the proof of the Lemma 1.

Lemma 2. Let $S$ be a sporadic simple group and let $P$ be a Sylow 2-subgroup of $S$. If $1 \neq x \in S$, then $S=\left\langle P, x^{g}\right\rangle$ for some $g \in S$.

Proof. Let $S$ be a sporadic simple group, fix a Sylow 2-subgroup $p$ of $S$, and let $x$ be a nonidentity element in $S$. We use known information about maximal subgroups of $S$ to show that $x^{S}$ is not a subset of the union of those maximal subgroups in $S$ that contain . Let $M$ be a maximal subgroup of $S$ with the property $P \leq M$. The number of $S$-conjugates of $M$ that contain $P$ is equal to $\left|N_{S}(P)\right| /\left|N_{M}(P)\right| \leq$ $\left[N_{S}(P): P\right]$, thus these subgroups cancontain at most $\left[N_{S}(P): P\right]\left|x^{S} \cap M\right|$ elements from the class $x^{S}$. Thus the number of elements in $x^{S}$ that generate a proper subgroup of $S$ together with $P$ is bounded from above by $\left[N_{S}(P): P\right] \Sigma_{M}\left|x^{S} \cap M\right|$, where the sum is taken over representatives $M$ of conjugacy classes of maximal subgroups of the odd index in $S$. Let $1_{M}^{S}$ denote the permutation character of $S$ on the cosets of . We have $\left|x^{S} \cap M\right|=\left|x^{S}\right| 1_{M}^{S}(x) / 1_{M}^{S}(1)$. Hence we are done when we show that

$$
\left[N_{S}(P): P\right] \Sigma_{M} 1_{M}^{S}(x) / 1_{M}^{S}(1)<1
$$

holds. The numbers $\left[N_{S}(P): P\right]$ can be read off from (Wilson, 1998). The Monster group is known to contain exactly five classes of maximal subgroups of odd index, of the structures $2^{1+24} \cdot C o_{1}, 2^{10+16} . O_{10}^{+}(2), 2^{2+11+22} \cdot\left(M_{24} \times\right.$ $\left.S_{3}\right), \quad 2^{5+10+20} .\left(S_{3} \times L_{5}(2)\right)$ and $\left[2^{39}\right] .\left(L_{3}(2) \times 3 S_{6}\right)$, also the corresponding permutation characters are known, see (Breuer, 2021). Since upper bound (1) is smaller than 1 , for each $x$ and $S$, we finished the proof.

Lemma 3. Let $S$ be a finite simply connected quasisimple group of Lie type in characteristic $p$. Let $P$ be a Sylow $p$-subgroup of $G$. If $x \in S$ is a noncentral element of $S$, then $S=\left\langle P, x^{g}\right\rangle$ for some $g \in S$.

Proof. By Tits's lemma (Seitz, 1973), we find that any maximal subgroup containing $P$ is a parabolic subgroup and there is precisely one parabolic subgroup containing $P$ in each conjugacy class of maximal parabolic subgroups. If $S$ has twisted rank 1, then the Borel subgroup is the unique maximal subgroup containing $T$ and so clearly no non-central conjugacy class is contained in the Borel subgroup, whence the result holds. So we may assume that the rank is at least 2. First, suppose that $S$ is a classical group. If $S=S L_{2}(q)$, then choose a basis for the natural module $v_{1}, \ldots, v_{n}$ and assume that $T$ stabilizes the flag associated with this ordered basis. Since $S$ acts 2-transitively on 1-dimensional spaces, given any non-central element $x \in S$, we can choose $g \in S$ so that $g^{-1} x g v_{1}$ is not in the span of $v_{1}, \ldots, v_{n-1}$ and so $x^{g}$ is not in any parabolic subgroup containing $T$. Now suppose $S$ is any other classical group with natural module $V$. Since we are assuming that $S$ has twisted rank at least $2, \operatorname{dim} V \geq 4$. First, suppose that $S$ is not an even-dimensional spin group in dimension at least 8. Fix $T$ a Sylow $p$-subgroup. Then the maximal parabolic subgroups correspond to totally singular spaces of distinct dimensions. Let $v_{1}$ be a nonzero element of the 1-dimensional fixed space of $T$. If $\mathrm{n}$ is the rank of $S$, then there is an ordered linearly independent set $v_{1}, \ldots, v_{n}$ so that any maximal parabolic subgroup containing $T$ is the stabilizer of the totally singular space $V_{i}=\left\langle v_{1}, \ldots, v_{i}\right\rangle$. Let $x$ be a non-central element of $S$. Suppose that $x v_{1}=w$. If $w$ and $v_{1}$ are linearly dependent, then just choose $g \in S$ so that $x^{g}$ maps $v_{1}$ to element not in $V_{n}$. If $w$ is not in $V_{n}$, then already $x$ is not in any of the parabolic subgroups containing $T$. The last case is if $\left\langle v_{1}, w\right\rangle$ is a 2-dimensional totally singular subspace of $V_{n}$. Then there exists $g \in S$ fixing $v_{1}$ and mapping $w$ to any singular vector in $v_{1}^{\perp}$ and since $v_{1}^{\perp}$ is not contained in $V_{n}$ (since $\operatorname{dim} V \geq 4$ ), the result holds in this case. Next, suppose that $S$ is an even-dimensional spin group of rank $n$. In the case, when we have an orthogonal module of dimension $2 n$, we choose a linearly independent set of basis vectors $v_{1}, \ldots, v_{n+1}$ so that the maximal parabolic subgroups containing $T$ are the stabilizer of the totally singular subspace $V_{i}=\left\langle v_{1}, \ldots, v_{i}\right\rangle, i=1, \ldots, n-2$ of dimension $i$ and the two totally singular subspaces $\left\langle v_{1}, \ldots, v_{n-1}, v_{n}\right\rangle$ and $\left\langle v_{1}, \ldots, v_{n-1}, v_{n+1}\right\rangle$. Finally, let us suppose that $S$ is an exceptional group of twisted 
rank $n$. Let $P_{1}, \ldots, P_{n}$ be the distinct maximal parabolic subgroups containing $T$. Let $C=x^{S}$ for $x$ a non-central element of $S$. It follows that (Lawther, Liebeck \& Seitz, 2002)

$$
\sum_{i=1}^{n} \frac{\left|C \cap P_{i}\right|}{|C|}<1
$$

Therefore $C \neq \bigcup_{i=1}^{n}\left(C \cap P_{i}\right)$, and therefore we finished the proof.

It remains to consider the special cases when $S$ is the simply connected group but $S / Z(S)$ is not simple. We can ignore the case when $S$ is a solvable group. This leaves the groups $S p_{4}(2), G_{2}(2),{ }^{3} G_{2}(3)$, and ${ }^{2} F_{4}(2)$. The first three cases have socle $\mathrm{Alt}_{6}, \mathrm{PSU}_{3}(3)$ and $P S L_{2}(8)$ and we have already proved the result for these groups. In the final case, the socle is the derived subgroup of index 2 but precisely the same previous argument (i.e. there are only two maximal subgroups containing a Sylow 2-subgroup which are parabolic subgroups intersected with the derived subgroup).

From previous lemmas, we reach the proof of the following fundamental theorem in this section.

Theorem 1. Let $S$ be a finite non-abelian simple group. Then there exists a prime $p_{1}$ with divides $|S|$, such that $S$ can be generated by a Sylow $p_{1}$-subgroup and a Sylow $p_{2}$-subgroup, where $p_{2}$ any given prime divides $|S|$.

\section{Generations of Finite Groups}

Recall that a subgroup $H$ of $G$ is called intravariant, if for any automorphism $\varphi$ of $G, \varphi(H)$ and $H$ are conjugate in $G$. The main result in this section will be presented in the following theorem.

Theorem 2. Let $p$ and $q$ are different primes and $G$ a finite group. Then there exists a Sylow $p$-subgroup $P$ of $G$ and an intravariant $q$-subgroup $R$ such that $G=\langle P, R\rangle$.

Proof: Let $p$ and $q$ be fixed primes, such that $p \neq q$, and let $G$ be a counterexample of minimal order. Firstly we will prove that: $O_{p}(G)=1$. Let us assume the converse and let $1 \neq A$ be a minimal characteristic subgroup of $G$ with $A$ an elementary abelian $p$-group. By the minimality, we find that $G / A=\langle P / A, R / A\rangle$, where $P$ is a Sylow $p$-subgroup of $G$ and $R$ is a subgroup of $G$ containing $A$ with $R / A$ an intravariant $q$-group of $G / A$. Since $\operatorname{gcd}(|R| /|A|,|A|)=1$, it follows that $H^{2}(R / A, A)=0$, therefore $R=A R_{1}$ with $R_{1}$ a complement of $A$ in $R$. In particular, $R_{1}$ is a $q$-subgroup. Suppose $a$ is an automorphism of $G$. Then by conjugating by an element of $G$, we may assume that $a(R)=R$. Thus, $a\left(R_{1}\right)$ is a complement of $A$ in $R$. Since $H^{1}\left(R_{1}, A\right)=0$, then all complements of $A$ in $R$ are conjugate via an element of $A$ and so $a\left(R_{1}\right)$ is conjugate to $R_{1}$, therefore $R_{1}$ is intravariant and it clear that $G=\left\langle P, R_{1}\right\rangle$.

At the second we will prove that: $O_{q}(G)=1$. Let us assume the converse and let $A$ be a minimal characteristic subgroup of $G$ with a $q$-subgroup $A$. We have $G / A=\langle Q / A, R / A\rangle$, where $Q / A$ is a Sylow $p$-subgroup of $G / A$ and $R / A$ is intravariant $q$-subgroup of $G / A$. Because $A$ is characteristic then $R$ is intravariant and clearly a $q$-subgroup. It is clear that $Q=A P$, where $P$ is a Sylow $p$-subgroup of $Q$ and of $G$. Thus $G=\langle P, R\rangle$ and the result follows.

Now, let $A$ be a minimal characteristic subgroup of $G$. By the previous arguments, we find that $A=S \times \ldots \times S$, where $S$ is a non-abelian simple group of order divisible by $p$. By Theorem 1 , we find that $A=\langle Q, M\rangle$, where $Q$ is a Sylow $p$-subgroup of $A$ and $M$ is a Sylow $r$-subgroup of $A$ for some $r \neq p$. Let $H=N_{G}(M)$, then $H$ is a proper subgroup of $G$, thus $H=\langle D, R\rangle$, where $D$ is a Sylow $p$-subgroup of $H$ and $R$ is an intravariant $q$-subgroup of $H$. Since $R$ normalizes $M$, we assume that $R \geq M$. $H$ is the normalizer of a Sylow subgroup of a characteristic subgroup of $G$ and so $H$ and $R$ are intravariant. Assume that $D$ contains a Sylow $p$-subgroup $Q_{1}$ of $A$. Then $Q_{1}^{a}=Q$ for some $a \in A$. Then $D^{a} \geq Q$. We have the following fact $G=J:=\left\langle D^{a}, R\right\rangle$. Firstly we have $J \geq\langle Q, M\rangle=A$. Since $a \in A$ we see that $D A=D^{a} A$ and so $J \geq\langle A, D, R\rangle \geq\langle A, H\rangle$. By the Frattini argument, we find that $G=H A$, thus $J=G$ as we need.

\section{References}

Aschbacher, M. (2004). The Status of the Classification of the Finite Simple Groups. Notices of the American Mathematical Society, 51(7), 736-740.

Aschbacher, M., \& Guralnick, R. (1984). Some applications of the first cohomology group. J. Algebra, 90, 446-460. https://doi.org/10.1016/0021-8693(84)90183-2

Breuer, T. (2021). Computations with the GAP Character Table Library. Retrieved from http://www.math.rwth-aachen.de/homes/sam/ctbllib/doc2/chap8_mj.html

Breuer, T., Guralnick, R., \& Kantor, W. (2008). Probabilistic generation of finite simple groups. ll, J. Algebra, 320, 443-494. https://doi.org/10.1016/j.jalgebra.2007.10.028

Burness, T. C., Guralnick, R. M., \& Harper, S. (2021). The spread of a finite group. Annals of Mathematics, 193(2), 619-687. https://doi.org/10.4007/annals.2021.193.2.5 
Burness, T. C., Liebeck, M. W., \& Shalev, A. (2013). Generation and random generation: from simple groups to maximal subgroups. Adv. Math., 248, 59-95. https://doi.org/10.1016/j.aim.2013.07.009

Dietrich, H., \& Low, D. (2021). Generation of finite groups with cyclic Sylow subgroups. J. Group Theory, 24, $161-175$. https://doi.org/10.1515/jgth-2020-0061

Gorenstein, D., Richard, L., \& Solomon, R. (2018). The Classification of the Finite Simple Groups, Number 8. MATHEMATICAL Surveys and Monographs, AMS. https://doi.org/10.1090/surv/040.8

King, C. S. (2017). Generation of finite simple groups by an involution and an element of prime order. J. Algebra, 478, 153-173. https://doi.org/10.1016/j.jalgebra.2016.12.031

Kurzweil, H., \& Stellmacher, B. (2004). The theory of finite groups: an introduction. Springer-Verlag New York, Inc. https://doi.org/10.1007/b97433

Lawther, R., Liebeck, M., \& Seitz, G. (2002). Fixed point ratios in actions of finite exceptional groups of Lie type. Pacific J. Math., 205, 393-464. https://doi.org/10.2140/pjm.2002.205.393

Martino, L. Di., Pellegrini, M., \& Zalesski, A. (2014). On generators and representations of the sporadic simple groups. Comm. Algebra, 42, 880-908. https://doi.org/10.1080/00927872.2012.729629

Praeger, C. E., \& Schneider, C. (2018). Permutation groups and Cartesian decompositions. London Mathematical Society Lecture Note Series 449. Cambridge: Cambridge University Press. https://doi.org/10.1017/9781139194006

Seitz, G. M. (1973). Flag-transitive subgroups of Chevalley groups. Ann. of Math., 97, $27-56$. https://doi.org/10.2307/1970876

Slattery, M. C. (2007). Generation of groups of square-free order. J. Symbolic Comput., 42(6), $668-677$. https://doi.org/10.1016/j.jsc.2007.01.006

Solomon, R. (2001). A Brief History of the Classification of the Finite Simple Groups. Bulletin of the American Mathematical Society, New Series, 38(3), 315-352. https://doi.org/10.1090/S0273-0979-01-00909-0

Steinberg, R. (1962). Generators for simple groups. Canad. J. Math., 14, $277-283$. https://doi.org/10.4153/CJM-1962-018-0

Wielandt, H. (1964). Finite permutation groups. Academic Press, New York-London.

Wilson, R. A. (1998). The McKay conjecture is true for the sporadic simple groups. J. Algebra, 207(1), $294-305$. https://doi.org/10.1006/jabr.1998.7450

\section{Copyrights}

Copyright for this article is retained by the author(s), with first publication rights granted to the journal.

This is an open-access article distributed under the terms and conditions of the Creative Commons Attribution license (http://creativecommons.org/licenses/by/4.0/). 